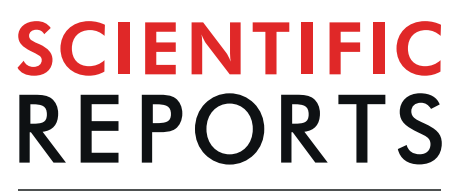

\title{
Association of immunophenotype with expression of topoisomerase II $\alpha$ and $\beta$ in adult acute myeloid leukemia
}

\author{
Andrew P. Michelson ${ }^{1}$, Shannon McDonough ${ }^{2}$, Cheryl L. Willman ${ }^{3}$, Eric R. Koegle ${ }^{1}$, \\ John E. Godwin ${ }^{4}$, Stephen H. Petersdorf ${ }^{5,9}$, Alan F. List ${ }^{6}$, Megan Othus ${ }^{2}$, \\ Frederick R. Appelbaum ${ }^{7}$, Jerald P. Radich ${ }^{7}$, Mahrukh K. Ganapathi ${ }^{8}$, Anjali S. Advani ${ }^{*}{ }^{\star}$ \& \\ Ram N. Ganapathi ${ }^{* *}$
}

Anthracyclines used in the treatment of acute myelogenous leukemia (AML) inhibit the activity of the mammalian topoisomerase II (topo II) isoforms, topo II $\alpha$ and topo II $\beta$. In 230 patients with non-M3 AML who received frontline ara-C/daunorubicin we determined expression of topo Il $\alpha$ and topo II $\beta$ by RTPCR and its relationship to immunophenotype (IP) and outcomes. Treatment outcomes were analyzed by logistic or Cox regression. In 211 patients, available for analysis, topo Il $\alpha$ expression was significantly lower than topo $I / \beta(P<0.0001)$. In contrast to topo II $\alpha$, topo II $\beta$ was significantly associated with blast percentage in marrow or blood $(P=0.0001), C D 7(P=0.01), C D 14(P<0.0001)$ and CD54 $(P<0.0001)$. Event free survival was worse for CD56-negative compared to CD56-high (HR=1.9, 95\% Cl [1.0-3.5], $\mathrm{p}=0.04)$, and overall survival was worse for $C D-15$ low as compared to $C D 15$-high $(\mathrm{HR}=2.2,95 \% \mathrm{Cl}$ [1.1-4.2], $p=0.02)$. Ingenuity pathway analysis indicated topo $\| \beta$ and immunophenotype markers in a network associated with cell-to-cell signaling, hematological system development/function and inflammatory response. Topo II $\beta$ expression reflects disease biology of highly proliferative disease and distinct IP but does not appear to be an independent variable influencing outcome in adult AML patients treated with anthracycline-based therapy.

Standard induction therapy for acute myeloid leukemia (AML) consists of a combination of cytarabine (ara-C) and an anthracycline, such as daunorubicin or idarubicin ${ }^{1,2}$. While ara-C is a pyrimidine analogue that prematurely terminates DNA polymerization, the anthracyclines are inhibitors of topoisomerase $\mathrm{II}^{3-6}$. DNA topoisomerases relieve the topological constraints associated with the double-helical structure of DNA during vital DNA metabolic processes, including DNA replication, transcription, chromosome segregation and recombination $^{7}$. Mammalian topoisomerase II (topo II) consists of two isoforms, topo II $\alpha\left(M_{r} 170 \mathrm{kDa}\right)$ and topo II $\beta\left(\mathrm{M}_{\mathrm{r}}\right.$ $180 \mathrm{kDa}$ ). Although the $\alpha$ and $\beta$ topo II isoforms are highly homologous and catalyze similar biochemical reactions, they are genetically distinct and exhibit different patterns of expression and cellular distribution ${ }^{7}$. While expression of topo II $\alpha$ is cell cycle-dependent ${ }^{7}$ topo II $\beta$ levels remain unchanged during cell cycle progression ${ }^{7-9}$ and are maximal in terminally differentiated tissues $8,10,11$. This difference in expression suggests that these two isoforms exert distinct functional roles in cellular processes that require topological changes in the DNA molecule. It has been suggested that topo II $\alpha$ may be important for DNA replication, whereas topo II $\beta$ may be involved in cellular differentiation ${ }^{8}$. Using stable expression of shRNA targeted to topo II $\alpha$ or topo II $\beta$, in cell culture models of human AML, we have been able to demonstrate a functional role for topo II $\beta$ in apoptosis following all-trans-retinoic acid induced differentiation ${ }^{12}$. Further, our studies have demonstrated that clinically active drugs

\footnotetext{
${ }^{1}$ Cleveland Clinic, Cleveland, OH, USA. ${ }^{2}$ SWOG Statistical Center, Seattle, WA, USA. ${ }^{3}$ University of New Mexico Cancer Research and Treatment Center, Albuquerque, NM, USA. ${ }^{4}$ Providence Onc/Hem Care Clinic, Portland, OR, USA. ${ }^{5}$ Seattle Genetics Inc, Bothell, WA, USA. ${ }^{6} \mathrm{H}$. Lee Moffitt Cancer Center \& Research Institute, Tampa, FL, USA. ${ }^{7}$ Fred Hutchinson Cancer Research Center, Seattle, WA, USA. ${ }^{8}$ Levine Cancer Institute, Carolinas Medical Center, Charlotte, NC, USA. ${ }^{9}$ Stephen H. Petersdorf is deceased. *email: advania@ccf.org; ganapar550@gmail.com
} 
target different isoforms of topo II to exert their anti-tumor activity and that topo II $\alpha$ and topo II $\beta$ cooperate to maintain genome stability, which may be modulated by their C-terminal domain ${ }^{13}$.

Although topo II is a putative target of daunorubicin that is a key component of the induction treatment regimen for AML for over 3 decades, the significance of topo II $\alpha$ and $\beta$ isoform expression and its association with other biomarkers related to outcome has not been satisfactorily addressed. Analysis of topo II $\alpha$ expression have suggested correlations between gene amplification of topo II $\alpha$ and response to anthracycline chemotherapy in breast cancer ${ }^{14}$. Some reports indicate no significant predictive value of topo II $\alpha$ expression levels, while others suggest that topo II $\alpha$ expression can predict treatment failure ${ }^{15-19}$. In contrast to the focus on topo II $\alpha$ in AML the expression of topo II $\beta$ that is targeted by daunorubicin and idarubicin has not been satisfactorily addressed. Topo II $\beta$ expression has been proposed to have a role in resistance to drugs that target this isoform ${ }^{20,21}$ and in resistance to all-trans retinoic acid (ATRA) induced differentiation in $\mathrm{M} 3 \mathrm{AML}^{22}$. Interestingly, mitoxantrone that targets topo II $\beta$ and other topo II inhibitors have been suggested to be involved in therapy related leukemia ${ }^{23}$. In the present study, we examined the expression of the topo II isoforms and possible relationships of topo II $\alpha$ and topo II $\beta$ expression to immunophenotype (IP) and outcomes in de novo and secondary adult AML blast samples from 230 patients enrolled in 4 SWOG studies who received ara-C/daunorubicin-based frontline chemotherapy.

Results

Correlation of topo II $\alpha$ and topo $I I \beta$ expression with clinical characteristics and immunophenotype markers. Of the 230 treatment-naïve specimens available, topo II expression data from 211 patients was available for analysis. Patient and clinical characteristics for the 211 patients are summarized in Table 1. Expression $(\Delta \mathrm{Ct})$ of the topo II isoforms was positively correlated (Fig. 1) and topo II $\beta$ expression was on average 2.2 -fold higher than topo II $\alpha$ expression (CI 1.8-2.6, $\mathrm{p}<0.001)$.

Association of topo II $\alpha$ or $\beta$ expression with clinical characteristics is outlined in Table 2. Topo II $\alpha$ expression was not found to be significantly associated with any patient or disease characteristic in univariate analyses. In contrast, topo II $\beta$ expression was inversely associated with age $(\mathrm{p}=0.001)$ and positively associated with both marrow and peripheral blast percentage $(\mathrm{p}<0.001)$. Topo II $\beta$ expression also varied significantly among FAB classes, being highest for M0 and lowest for M4 and M7 ( $=0.0012)$. Since immunophenotype is associated with outcome, analysis of the correlation of topo II $\alpha$ or $\beta$ expression with IP markers was carried out and is outlined in Table 3. Topo II $\alpha$ expression was not correlated with any of the immunophenotypic markers measured. In contrast, topo II $\beta$ expression was associated with expression of CD4 ( $p=0.0025), C D 7(p=0.01), C D 11 a$ $(\mathrm{p}<0.0001), \operatorname{CD} 11 \mathrm{~b}(\mathrm{p}<0.0001), \operatorname{CD} 11 \mathrm{c}(\mathrm{p}=0.007), \operatorname{CDw} 14(\mathrm{p}<0.0001), \operatorname{CD} 15(\mathrm{p}=0.045), \operatorname{CD} 16(\mathrm{p}=0.009)$, CD34 ( $\mathrm{p}=0.03)$, CD54 ( $<<0.0001)$ and HLA-DR $(\mathrm{p}<0.0003)$. In multivariate analysis of 137 patients with complete data, topo II $\beta$ expression was positively associated with age, as well as blood and marrow blast percentage $(\mathrm{p}<0.001)$. Additional regression analysis of IP with outcome measures revealed event free survival was worse for CD56-ve compared to CD56-high ( $\mathrm{HR}=1.9,95 \% \mathrm{CI}[1.0-3.5], \mathrm{p}=0.04)$, and overall survival was worse for $\mathrm{CD} 15$-low as compared to CD15-high $(\mathrm{HR}=2.2,95 \% \mathrm{CI}[1.1-4.2], \mathrm{p}=0.02)$. Neither topo II $\alpha$ expression nor topo II $\beta$ expression was significantly associated with clinical outcomes (CR, RD, RFS and OS) in uni- or multivariate analysis.

Data from analysis of P-glycoprotein expression and function, representing possible determinants of resistance was available and correlation with topo II isoform expression was assessed. Topo II $\beta$ expression was weakly correlated with P-glycoprotein expression detected with the MM4.17 antibody $(\mathrm{p}=0.07)$ and P-glycoprotein function based on rhodamine efflux $(\mathrm{p}=0.04)$. Both parameters were inversely related to topo II $\beta$ expression but not correlated with topo II $\alpha$ expression. However, in univariate analysis neither P-glycoprotein expression or function was associated with OS, RFS or EFS.

Pathway analysis of immunophenotype markers with topo II $\alpha$ and topo II $\beta$. Ingenuity pathway analysis of drug pharmacodynamic targets, immunophenotype markers and topo II are outlined in Figs. 2 and 3. In Fig. 2, among 6 networks the major network with a score of 34 and 15 focus molecules identified the direct interaction of topo II $\alpha /$ topo II $\beta$ and cell-to-cell signaling, hematological system development/function and immune cell trafficking as the top diseases and functions. In Fig. 3, with a focus on topo II $\beta$ and the significantly associated immunophenotype markers, among 3 networks the major network generated a score of 25 with 9 focus molecules and an association with cell-to-cell signaling/interaction, hematological system development/function and inflammatory response among the top diseases and functions. Overall, both networks identified similar functional events and disease states based on the interaction between significantly associated immunophenotype markers and topo II.

\section{Discussion}

This is the first study examining the expression levels of both topo II $\alpha$ and II $\beta$ in a large cohort of de novo and secondary AML patients and evaluating associations between topo II isoform expression, clinical outcome, immunophenotype and other patient characteristics. Inhibitors of topo II, such as the anthracyclines, are the cornerstones of AML treatment and presumed primarily to target topo II $\alpha$. Despite the importance of the anthracyclines at inducing complete remission, it is unknown if the expression levels of topo II $\alpha$ can predict clinical outcome. Present results do demonstrate a significant inter-individual variability in topo II $\alpha$ mRNA levels and failed to show any significant association between topo II $\alpha$ expression and any disease characteristic in de novo and secondary AML patients. It has been shown that exposure of AML blast cells to the anthracyline daunorubicin promotes expansion of topo II $\alpha$ negative cells ${ }^{19}$. This ex-vivo observation, on daunorubicin-treatment dependent selection of topo II $\alpha$ negative cells was however, not linked to clinical outcome. Despite active investigation into the clinical significance of topo II $\alpha$, little is known about the importance of topo II $\beta$ in AML. Gieseler et al. ${ }^{24}$ reported that blast cells from patients with elevated activity of topo II $\beta$ expressed reduced sensitivity in vitro to daunorubicin or 


\begin{tabular}{|c|c|c|}
\hline Characteristics & Median & Range \\
\hline Age (yrs) & 64 & $19-88$ \\
\hline Marrow blasts (\%) & 70 & $4-99$ \\
\hline WBC $\left(10^{9} / \mathrm{L}\right)$ & 29.6 & $0.8-274$ \\
\hline Peripheral blasts (\%) & 38 & $0-99$ \\
\hline Hemoglobin (g/dL) & 9.1 & $4.3-13.7$ \\
\hline \multirow[t]{2}{*}{ Platelets $\left(10^{9} / \mathrm{L}\right)$} & 58.5 & $2-1052$ \\
\hline & Number & $\%$ \\
\hline \multicolumn{3}{|l|}{ Sex: } \\
\hline Female & 94 & 45 \\
\hline Male & 117 & 55 \\
\hline \multicolumn{3}{|l|}{ Race/Ethnicity: } \\
\hline Native American & 1 & 0.5 \\
\hline Asian, Pacific Islander & 7 & 3 \\
\hline Black, African American & 17 & 8 \\
\hline White/Hispanic & 5 & 2 \\
\hline White/Non-Hispanic & 179 & 85 \\
\hline Hispanic, NOS & 2 & 1 \\
\hline \multicolumn{3}{|l|}{ AML Onset (months): } \\
\hline De Novo & 170 & 81 \\
\hline Secondary & 41 & 19 \\
\hline \multicolumn{3}{|l|}{ FAB Class (local diagnosis): } \\
\hline M1 & 47 & 22 \\
\hline M2 & 78 & 37 \\
\hline M4 & 46 & 22 \\
\hline M5 & 22 & 10 \\
\hline M6 & 1 & 0.5 \\
\hline M7 & 2 & 1 \\
\hline M0 & 9 & 4 \\
\hline Other & 5 & 2 \\
\hline \multicolumn{3}{|l|}{ Performance Status } \\
\hline 0 & 63 & 30 \\
\hline 1 & 104 & 49 \\
\hline 2 & 25 & 12 \\
\hline 3 & 17 & 8 \\
\hline Unknown & 2 & 1 \\
\hline \multicolumn{3}{|l|}{ Cytogenetics Evaluated: } \\
\hline Yes & 169 & 80 \\
\hline No & 42 & 20 \\
\hline \multicolumn{3}{|l|}{ Karyotype Category:* } \\
\hline Normal & 78 & 46 \\
\hline $\mathrm{t}(8: 21)$ & 7 & 4 \\
\hline $\operatorname{inv}(16) / t(16 ; 16)$ & 7 & 4 \\
\hline$-5 / \operatorname{del}(5 q)$ & 7 & 4 \\
\hline$-7 / \operatorname{del}(7 q)$ & 9 & 5 \\
\hline Other & 9 & 5 \\
\hline \multicolumn{3}{|l|}{ Study } \\
\hline S9031 & 75 & 36 \\
\hline S9126 & 10 & 5 \\
\hline S9333 & 81 & 38 \\
\hline S9500 & 45 & 21 \\
\hline
\end{tabular}

Table 1. Characteristics of 211 adult patients with previously untreated $(\mathrm{N}=211)$ non-M3-AML. *Percentages for karyotype categories are based on patients with cytogenetics evaluated.

idarubicin and relapse from treatment with anthracyclines may be linked to a significantly lower topo II $\alpha / \beta$ ratio. Our studies in HL-60 cells with targeted stable down-regulation of topo II $\alpha$ or $\beta$ isoform or in models engineered to express either topo II $\alpha$ or topo II $\beta$, indicate that while sensitivity to doxorubicin is unaltered, a 2 - to 4 -fold reduction in etoposide sensitivity is observed following down-regulation of the $\alpha$ isoform, and a marked decrease in sensitivity to amsacrine, idarubicin and mitoxantrone is seen in cells depleted of the $\beta$ isoform ${ }^{12,13}$. However, 


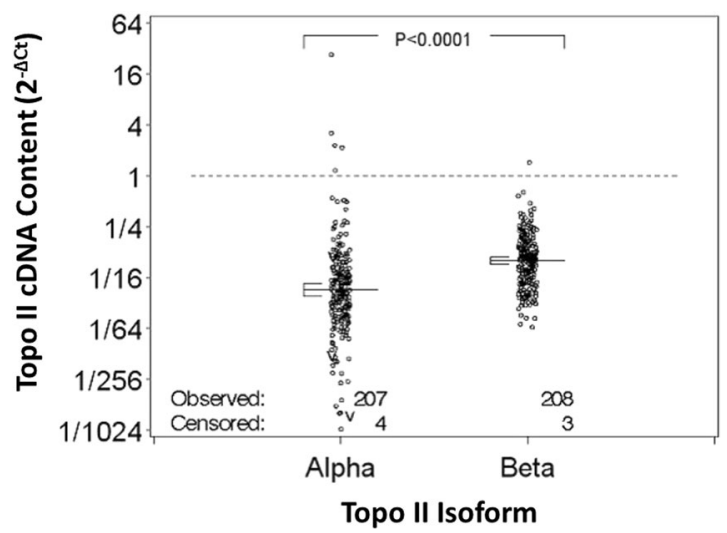

Figure 1. Topoisomerase II expression by topo II isoform in pre-treatment marrow specimens from 211 adult AML patients. The delta $\mathrm{Ct}(\Delta \mathrm{Ct})$ was calculated by subtracting the average cycle threshold $(\mathrm{Ct})$ for each topo II isoform from the average $\beta_{2}$-microglobulin (Ct). " $\mathrm{V}$ " indicates censored data. Solid lines show mean relative expression $(\Delta \mathrm{Ct})$ and $95 \%$ confidence interval. Mean relative expression was 2.2 -fold higher for topo II $\beta$ compared to topo II $\alpha$.

topo II $\beta$ was significantly associated with several factors that have been associated with favorable outcomes in AML, such as younger age, low CD4, CD14, CD16, CD54, CD11b, and HLA-DR, as well as with unfavorable factors, high peripheral and marrow blast percentage and increased CD7 expression. While reports on relationship or precise role of immunophenotype and prognosis in AML is controversial ${ }^{25-29}$, the association with topo II $\beta$ but not topo II $\alpha$ expression and proposed network of topo II $\beta$ with immunophenotype markers suggests a potential role for topo II $\beta$ expression and immunophenotype in the biology of AML. Song et al.$^{30}$ reported high topo II $\beta /$ topo II $\alpha$ expression to be correlative with favorable outcome but this observation could not be compared with the present results since induction therapy utilized the anthracycline idarubicin and most patients also received hematopoietic stem cell transplantation. Recent reports ${ }^{31,32}$ on use of idarubicin in adult AML indicate: (a) that an increased cumulative dose of idarubicin during consolidation can improve leukemia-free survival; and (b) comparing idarubicin to high dose daunorubicin during induction did not indicate significant differences in CR rate, relapse and survival. Heterogeneity in development of resistant cells as well as differential expression in key pathways has been suggested to involved in refractory $\mathrm{AML}^{33,34}$. Analysis of topo II $\alpha$ and $\beta$ expression coupled with immunophenotype in AML cells from patients with disease that is resistant to anthracycline/cytarabine therapy might provide insights on biomarkers relevant to outcome. In summary, topo II $\beta$ expression reflects aspects of disease biology, such as highly proliferative disease (higher blasts) and immunophenotypic differences but does not appear to be an independent variable influencing outcome in adult AML patients treated with anthracycline-based therapy.

\section{Materials and Methods}

Patients and specimens. Bone marrow (BM) specimens were provided by the SWOG AML/MDS Repository for 230 adult patients with non-M3 AML by FAB criteria who were enrolled during 1992-1998 for ara-C/daunorubicin-based frontline chemotherapy on any of four SWOG studies S9031, S9126, S9333 and S9500. Specimens of cryopreserved BM cells and expected to contain $>70 \%$ blasts, were used when available, otherwise RNA extracted from specimens upon receipt at the repository were used. All patients provided written informed consent in accordance with institutional and federal guidelines. The protocol was approved by the Cleveland Clinic IRB CC 937: S9031-S9126-S9333-S9500-B Topoisomerase 2 Expression and Acute Myeloid Leukemia (AML).

The study was performed in accordance with the Declaration of Helsinki.

RNA extraction. RNA extraction was performed on the entire BM specimen using the Trizol reagent (Invitrogen USA) per the manufacturer's instructions. The RNA pellets were re-suspended in $50 \mu \mathrm{L}$ RNase-free water and stored at $-80^{\circ} \mathrm{C}$.

qRT-PCR. RNA (1500 ng) was reverse transcribed to cDNA and used for quantitative PCR reaction, which was carried out in triplicate at the Case Comprehensive Cancer Center, Gene Expression Core Facility using an ABI PRISM 7900HT (Martina Veigl, Director). The primers used for the PCR reaction were: topo II $\alpha$ (forward: 5'-TGTCTCTCAAAAGCCTGATC-3' ${ }^{\prime}$, reverse: $5^{\prime}$-GTCCATATGGAAGTCATCAC-3'), topo II $\beta$ (forward: 5'-TAAAGGCCGAGGGGCAAAGA-3', reverse: 5'-GCAGAGAAGGTGGCTCAGTA-3') specific primers and $\beta_{2}$-microglobulin (B2MG) (forward: $5^{\prime}$-CTTGTCTTTCAGCAAGGACTGG-3' and reverse: 5'-CATGATGCTGCTTACATGTCTC-3'primers). B2MG was used as an endogenous control to normalize topo II $\alpha$ and topo II $\beta$ expression.

Immunophenotypic and cytogenetic analysis. Immunophenotyping was performed at the SWOG AML/MDS Repository at the University of New Mexico (Cheryl Willman, Director). Blast cells were assessed for expression, of the following IP markers: CD2, CD4, CD7, CD8, CD11a, CD11b, CD11c, CD13, CDW14, CD15, CD16, CD18, CD19, CD33, CD34, CD38, CD44, CD54, CD56 and HLA-DR. Expression of these markers in 


\begin{tabular}{|c|c|c|c|c|c|c|c|c|}
\hline & & \multirow[b]{2}{*}{ Pts. } & \multicolumn{3}{|c|}{ Topo II $\Delta \mathrm{Ct}$} & \multicolumn{3}{|c|}{ Topo II $\beta \Delta \mathrm{Ct}$} \\
\hline & & & ${ }^{\mathrm{a}}$ Mean & $95 \% \mathrm{CI}$ & P* & $\begin{array}{l}\text { a Mean } \\
\end{array}$ & $95 \% \mathrm{CI}$ & $\mathrm{P}^{*}$ \\
\hline \multirow[t]{2}{*}{ Sex } & F & 94 & 4.6263 & $(4.2279,5.0248)$ & 0.26 & 3.3033 & $(3.0780,3.5287)$ & 0.62 \\
\hline & M & 117 & 4.3317 & $(3.9978,4.6656)$ & & 3.3768 & $(3.1880,3.5656)$ & \\
\hline \multirow[t]{5}{*}{ Race } & Asian & 7 & 3.4503 & $(0.8010,6.0996)$ & 0.52 & 4.1253 & $(2.8630,5.3877)$ & 0.25 \\
\hline & Black & 17 & 4.6634 & $(4.0769,5.2499)$ & & 3.5518 & $(3.0479,4.0557)$ & \\
\hline & N.A./A.A.** & 1 & 4.5682 & NA & & 3.3044 & NA & \\
\hline & White & 184 & 4.4968 & $(4.2205,4.7730)$ & & 3.3020 & $(3.1487,3.4553)$ & \\
\hline & Unknown & 2 & 3.1383 & $(-2.9872,9.2638)$ & & 2.7353 & $(-2.7070,8.1776)$ & \\
\hline \multirow[t]{2}{*}{ Hispanic } & No & 204 & 4.4853 & $(4.2234,4.7472)$ & 0.35 & 3.3421 & $(3.1947,3.4895)$ & 0.88 \\
\hline & Yes & 7 & 3.8123 & $(2.7032,4.9213)$ & & 3.4018 & $(2.5783,4.2252)$ & \\
\hline Secondary & No & 170 & 4.4411 & $(4.1584,4.7238)$ & 0.73 & 3.2957 & $(3.1359,3.4555)$ & 0.18 \\
\hline AML & Yes & 41 & 4.5534 & $(3.9369,5.1700)$ & & 3.5445 & $(3.2064,3.8826)$ & \\
\hline SWOG & S9031 & 75 & 4.7364 & $(4.4125,5.0603)$ & 0.19 & 3.3004 & $(3.0788,3.5220)$ & $<0.0001$ \\
\hline \multirow[t]{3}{*}{ trial } & S9126 & 10 & 5.1018 & $(3.5095,6.6940)$ & & 4.1963 & $(3.5343,4.8583)$ & \\
\hline & S9333 & 81 & 4.3295 & $(3.8460,4.8130)$ & & 3.5950 & $(3.3656,3.8245)$ & \\
\hline & S9500 & 45 & 4.1055 & $(3.5477,4.6632)$ & & 2.7758 & $(2.4663,3.0852)$ & \\
\hline Year of & 1992 & 32 & 4.8509 & $(4.4034,5.2984)$ & 0.24 & 3.4673 & $(3.1730,3.7616)$ & 0.076 \\
\hline entry & 1993 & 35 & 4.6456 & $(4.0938,5.1973)$ & & 3.1022 & $(2.7214,3.4831)$ & \\
\hline into & 1994 & 11 & 5.2779 & $(4.0656,6.4903)$ & & 3.9856 & $(3.2767,4.6945)$ & \\
\hline \multirow[t]{4}{*}{ trial } & 1995 & 20 & 4.2586 & $(3.4299,5.0874)$ & & 3.4189 & $(2.8697,3.9680)$ & \\
\hline & 1996 & 51 & 3.9532 & $(3.3684,4.5380)$ & & 3.2415 & $(2.9486,3.5344)$ & \\
\hline & 1997 & 42 & 4.4320 & $(3.9085,4.9554)$ & & 3.1622 & $(2.8130,3.5114)$ & \\
\hline & 1998 & 20 & 4.6438 & $(3.3084,5.9791)$ & & 3.7860 & $(3.3334,4.2387)$ & \\
\hline FAB & M0 & 9 & 4.5263 & $(2.6133,6.4393)$ & 0.85 & 2.4874 & $(1.6527,3.3221)$ & 0.0012 \\
\hline class & M1 & 48 & 4.3428 & $(3.9809,4.7048)$ & & 3.0910 & $(2.8157,3.3664)$ & \\
\hline (local & M2 & 78 & 4.3830 & $(3.9286,4.8374)$ & & 3.2699 & $(3.0202,3.5196)$ & \\
\hline \multirow[t]{5}{*}{ diagnosis) } & M4 & 46 & 4.6417 & $(3.9839,5.2995)$ & & 3.7800 & $(3.4969,4.0630)$ & \\
\hline & M5 & 22 & 4.2870 & $(3.5581,5.0158)$ & & 3.6301 & $(3.2513,4.0088)$ & \\
\hline & M6 & 1 & 5.6905 & NA & & 3.0522 & NA & \\
\hline & M7 & 2 & 4.1100 & $(3.0501,5.1699)$ & & 3.7085 & $(-0.892,7.5062)$ & \\
\hline & Other & 5 & 5.2328 & $(2.6278,7.8377)$ & & 3.5783 & $(1.8490,5.3077)$ & \\
\hline Cytogenetic & Favorable & 14 & 4.2439 & $(2.6233,5.8644)$ & 0.66 & 3.7951 & $(3.1936,4.3965)$ & 0.34 \\
\hline \multirow[t]{4}{*}{ risk group } & Int-Normal & 78 & 4.3503 & $(3.8822,4.8184)$ & & 3.2937 & $(3.0178,3.5696)$ & \\
\hline & Int-II & 51 & 4.7509 & $(4.3165,5.1852)$ & & 3.4822 & $(3.2511,3.7132)$ & \\
\hline & Unfavorable & 26 & 4.4781 & $(3.9370,5.0193)$ & & 3.2704 & $(3.8841,3.6566)$ & \\
\hline & & Pts & ${ }^{\mathrm{b} C o e f f .}$ & $95 \% \mathrm{CI}$ & P* & ${ }^{\mathrm{b} C o e f f .}$ & $95 \% \mathrm{CI}$ & P* \\
\hline Age (years) & & 211 & 0.00633 & $(-0.0112,0.0239)$ & 0.48 & 0.0163 & $(0.0067,0.0260)$ & 0.001 \\
\hline Marrow blasts (\%) & & 196 & -0.00167 & $(-0.0141,0.0108)$ & 0.79 & -0.0165 & $(-0.0230,-0.0101)$ & $<0.0001$ \\
\hline $\operatorname{WBC}\left(10^{9} / \mathrm{L}\right)$ & & 211 & 0.00089 & $(-0.0041,0.0059)$ & 0.73 & -0.0009 & $(-0.0037,0.0020)$ & 0.55 \\
\hline Peripheral blasts (\%) & & 202 & 0.00054 & $(-0.0079,0.0090)$ & 0.90 & -0.0123 & $(-0.0167,-0.0078)$ & $<0.0001$ \\
\hline Hemoglobin $(\mathrm{g} / \mathrm{dL})$ & & 206 & 0.01830 & $(-0.1297,0.1663)$ & 0.81 & -0.0048 & $(-0.0876,0.0781)$ & 0.91 \\
\hline Platelets $\left(10^{9} / \mathrm{L}\right)$ & & 210 & 0.00157 & $(-0.0011,0.0042)$ & 0.25 & 0.0016 & $(0.0001,0.0031)$ & \begin{tabular}{|l|}
0.04 \\
\end{tabular} \\
\hline
\end{tabular}

Table 2. Topo II $\alpha$ and II $\beta$ expression by clinical characteristics of 211 treatment-naïve adult non-M3 AML patients. Abbreviations: Pts. = patients; $\mathrm{CI}=$ confidence interval. ${ }^{\mathrm{a}}$ Means (and $95 \% \mathrm{CI}$ of the means), represent

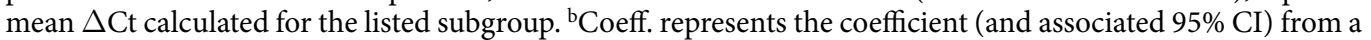
univariate linear regression model with the $\Delta \mathrm{Ct}$ variable as the outcome and the listed variables as covariates. Coeff. greater than 0 indicates a positive association: as the covariate values increase, the $\Delta \mathrm{Ct}$ values increase on average. Coeff. less than 0 indicates a negative association; as the covariate values increase, the $\Delta \mathrm{Ct}$ values decrease on average. *P-value for heterogeneity of mean $\Delta \mathrm{Ct}$ among categories or regression on continuous variables; calculated from univariate linear regression models with $\Delta \mathrm{Ct}$ as the outcome and each variable as a covariate. **N.A./A.A.: Native America or Alaskan Native.

the total population was characterized as negative, high, low, low/high or low/negative. Four other markers were assayed but excluded from this analysis: CD3, CD8 were negative for all patients; CD38 and CD45 were highly expressed in all but 2 patients. Cytogenetic studies of pretreatment marrow or peripheral blood were performed at SWOG-approved laboratories and centrally reviewed by the SWOG Cytogenetics Committee.

Statistical and pathway analysis. The $\Delta \mathrm{Ct}$ for topo II $\alpha$ or II $\beta$ RNA was calculated by subtracting the average cycle threshold (Ct) for each topo II isoform from the average $\beta_{2}$-microglobulin (Ct). Average topo II 


\begin{tabular}{|c|c|c|c|c|c|c|c|c|}
\hline \multirow{2}{*}{\multicolumn{2}{|c|}{ Immunophenotype * }} & \multirow{3}{*}{$\begin{array}{l}\text { Pts. } \\
10\end{array}$} & \multicolumn{3}{|c|}{ Topo II $\Delta \mathrm{Ct}$} & \multicolumn{3}{|c|}{ Topo II $\beta \Delta \mathrm{Ct}$} \\
\hline & & & \multirow{2}{*}{\begin{tabular}{|l|} 
Mean \\
4.2453 \\
\end{tabular}} & \multirow{2}{*}{\begin{tabular}{|l|}
$\mathbf{9 5} \%$ CI \\
$(3.3954,5.0952)$ \\
\end{tabular}} & \multirow{2}{*}{\begin{tabular}{|l|} 
P*** \\
0.67 \\
\end{tabular}} & \multirow{2}{*}{\begin{tabular}{|l|} 
Mean \\
2.8719 \\
\end{tabular}} & \multirow{2}{*}{$\begin{array}{l}\mathbf{9 5 \%} \mathrm{CI} \\
(1.9956,3.7486)\end{array}$} & \multirow{2}{*}{\begin{tabular}{|r|}
$\mathbf{P} * *$ \\
0.14 \\
\end{tabular}} \\
\hline $\mathrm{CD} 2$ & HIGH/LOW & & & & & & & \\
\hline & NEGATIVE & 176 & 4.5147 & $(4.2180,4.8114)$ & & 3.3844 & $(3.2255,3.5432)$ & \\
\hline \multirow[t]{3}{*}{$\mathrm{CD} 4$} & HIGH & 16 & 4.1362 & $(3.2256,5.0468)$ & 0.50 & 3.7466 & $(3.3509,4.1423)$ & 0.0025 \\
\hline & LOW & 41 & 4.7695 & $(4.2694,5.2695)$ & & 3.7716 & $(3.4116,4.1316)$ & \\
\hline & NEGATIVE & 129 & 4.4598 & $(4.0953,4.8242)$ & & 3.1766 & $(2.9935,3.3597)$ & \\
\hline \multirow[t]{3}{*}{$\mathrm{CD} 7$} & HIGH & 23 & 3.9183 & $(3.1032,4.7334)$ & 0.27 & 2.8087 & $(2.4433,3.1742)$ & 0.010 \\
\hline & LOW & 21 & 4.8258 & $(4.1192,5.5324)$ & & 3.6811 & $(3.2225,4.1396)$ & \\
\hline & NEGATIVE & 140 & 4.5218 & $(4.1847,4.8589)$ & & 3.4168 & $(3.2357,3.5978)$ & \\
\hline \multirow[t]{3}{*}{ CD11A } & HIGH & 109 & 4.4966 & $(4.1603,4.8328)$ & 0.57 & 3.6105 & $(3.4184,3.8027)$ & 0.0002 \\
\hline & LOW & 48 & 4.3216 & $(3.6616,4.9816)$ & & 3.1290 & $(2.8229,3.4351)$ & \\
\hline & NEGATIVE & 29 & 4.8095 & $(4.0099,5.6091)$ & & 2.7802 & $(2.3649,3.1954)$ & \\
\hline \multirow[t]{3}{*}{ CD11B } & HIGH & 41 & 4.8756 & $(4.3956,5.3556)$ & 0.30 & 4.0955 & $(3.8647,4.3263)$ & $<0.0001$ \\
\hline & LOW & 25 & 4.6374 & $(3.8819,4.7239)$ & & 3.0996 & $(2.7450,3.4541)$ & \\
\hline & NEGATIVE & 120 & 4.3434 & $(3.9628,2.9839)$ & & 3.1580 & $(2.9567,3.3593)$ & \\
\hline \multirow[t]{3}{*}{ CD11C } & HIGH & 94 & 4.5009 & $(4.1292,4.8727)$ & 0.88 & 3.5975 & $(3.3765,3.8185)$ & 0.007 \\
\hline & LOW & 48 & 4.3987 & $(3.7782,5.0193)$ & & 3.0564 & $(2.7458,3.3669)$ & \\
\hline & NEGATIVE & 44 & 4.6093 & $(3.9745,5.2442)$ & & 3.1704 & $(2.8697,3.4710)$ & \\
\hline \multirow[t]{3}{*}{$\mathrm{CD} 13$} & HIGH & 134 & 4.3263 & $(3.9917,4.6609)$ & 0.12 & 3.3823 & $(3.2031,3.5615)$ & 0.42 \\
\hline & LOW & 34 & 5.0728 & $(4.4835,5.6621)$ & & 3.4227 & $(3.0434,3.8019)$ & \\
\hline & NEGATIVE & 18 & 4.7135 & $(3.5730,5.8540)$ & & 3.0426 & $(2.4036,3.6815)$ & \\
\hline \multirow[t]{3}{*}{ CDw14 } & HIGH & 34 & 4.7128 & $(4.0369,5.3887)$ & 0.55 & 4.2883 & $(4.0132,4.5633)$ & $<0.0001$ \\
\hline & LOW & 17 & 4.8316 & $(3.9607,5.7026)$ & & 3.7438 & $(3.2190,4.2687)$ & \\
\hline & NEGATIVE & 135 & 4.4049 & $(4.0647,4.7451)$ & & 3.0735 & $(2.9024,3.2445)$ & \\
\hline \multirow[t]{3}{*}{ CD15 } & HIGH & 97 & 4.4044 & $(4.0045,4.8042)$ & 0.79 & 3.5449 & $(3.3310,3.7589)$ & 0.045 \\
\hline & LOW & 23 & 4.5911 & $(3.8205,5.3617)$ & & 3.1579 & $(2.7509,3.5649)$ & \\
\hline & NEGATIVE & 66 & 4.6094 & $(4.1177,5.1010)$ & & 3.1497 & $(2.8760,3.4233)$ & \\
\hline CD16 & HIGH/LOW & 7 & 5.8795 & $(4.2576,7.5014)$ & 0.06 & 4.3967 & $(3.4498,5.3454)$ & 0.009 \\
\hline & NEGATIVE & 179 & 4.4463 & $(4.1585,4.7340)$ & & 3.3161 & $(3.1593,3.4729)$ & \\
\hline CD18 & HIGH & 121 & 4.3574 & $(4.0092,4.7057)$ & 0.06 & 3.4947 & $(3.3033,3.6860)$ & 0.058 \\
\hline & LOW & 34 & 4.3074 & $(3.5520,5.0629)$ & & 3.0955 & $(2.7007,3.4902)$ & \\
\hline & NEGATIVE & 30 & 5.2622 & $(4.6282,5.8963)$ & & 3.0977 & $(2.7122,3.4831)$ & \\
\hline CD19 & HIGH/LOW & 6 & 5.2026 & $(3.2847,7.1204)$ & 0.37 & 3.4779 & $(2.4885,4.4673)$ & 0.78 \\
\hline & NEGATIVE & 180 & 4.4768 & $(4.1881,4.7655)$ & & 3.3528 & $(3.1931,3.5124)$ & \\
\hline CD33 & HIGH & 159 & 4.4490 & $(4.1326,4.7654)$ & 0.59 & 3.3184 & $(3.1443,3.4925)$ & 0.50 \\
\hline & LOW & 23 & 4.7134 & $(4.0052,5.4216)$ & & 3.5973 & $(3.2237,3.9708)$ & \\
\hline & NEGATIVE & 4 & 5.3101 & $(4.0324,6.5878)$ & & 3.5012 & $(2.1531,4.8492)$ & \\
\hline CD34 & HIGH & 106 & 4.6027 & $(4.2370,4.9685)$ & 0.07 & 3.5299 & $(3.3438,3.7159)$ & 0.03 \\
\hline & LOW & 6 & 2.6970 & $(0.6967,4.6972)$ & & 2.7758 & $(1.5067,4.0448)$ & \\
\hline & NEGATIVE & 74 & 4.4996 & $(4.0361,4.9630)$ & & 3.1560 & $(2.8823,3.4298)$ & \\
\hline CD44 & HIGH & 182 & 4.4889 & $(4.2018,4.7760)$ & 0.74 & 3.3482 & $(3.1893,3.5072)$ & 0.40 \\
\hline & LOW/NEGATIVE & 3 & 4.8630 & $(-0.9289,10.655)$ & & 3.8840 & $(2.2043,5.5638)$ & \\
\hline CD54 & HIGH-SUB & 11 & 4.8624 & $(4.2678,5.4571)$ & 0.32 & 3.8084 & $(3.4275,4.1893)$ & $<0.0001$ \\
\hline & HIGH-TOT & 47 & 4.8973 & $(4.3107,5.4840)$ & & 3.5599 & $(3.2425,3.8773)$ & \\
\hline & LOW-TOT & 64 & 4.2672 & $(3.7429,4.7916)$ & & 3.6311 & $(3.3984,3.8638)$ & \\
\hline & NEGATIVE & 63 & 4.3619 & $(3.8810,4.8428)$ & & 2.8482 & $(2.5649,3.1315)$ & \\
\hline CD56 & HIGH & 27 & 4.6836 & $(3.8938,5.4734)$ & 0.60 & 3.6942 & $(3.2065,4.1818)$ & 0.20 \\
\hline & LOW & 5 & 3.7128 & $(0.1725,7.2530)$ & & 3.4801 & $(2.1129,4.8474)$ & \\
\hline & NEGATIVE & 154 & 4.4936 & $(4.1865,4.8007)$ & & 3.2937 & $(3.1272,3.4601)$ & \\
\hline HLA-DR & HIGH & 154 & 4.4355 & $(4.1422,4.7287)$ & 0.58 & 3.4844 & $(3.3177,3.6511)$ & 0.0003 \\
\hline & LOW & 5 & 5.0998 & $(2.3465,7.8532)$ & & 3.5153 & $(2.3476,4.6829)$ & \\
\hline & NEGATIVE & 27 & 4.7585 & $(3.7570,5.7600)$ & & 2.5996 & $(2.2039,2.9952)$ & \\
\hline
\end{tabular}

Table 3. Topo II $\alpha$ and II $\beta$ expression by immunophenotype markers of 186 treatment-naïve adult non-M3 AML patients. Abbreviations: Pts = patients; $\mathrm{CI}=$ confidence interval. *Expression of immunophenotype markers was originally classified as high in the total blast population (HIGH-TOT) or a subpopulation (HIGH-SUB), low in the total blast population (LOW-TOT) or a subpopulation (LOW-SUB), or negative. These categories were combined as needed to ensure adequate category sizes for comparisons when possible. **P-value for heterogeneity of mean $\Delta \mathrm{Ct}$ among immunophenotype categories calculated from univariate linear regression models with $\Delta \mathrm{Ct}$ as the outcome and each immunophenotype marker as the covariate; means and $95 \% \mathrm{CIs}$ of the means of $\Delta \mathrm{Ct}$ were calculated for each subgroup listed. 


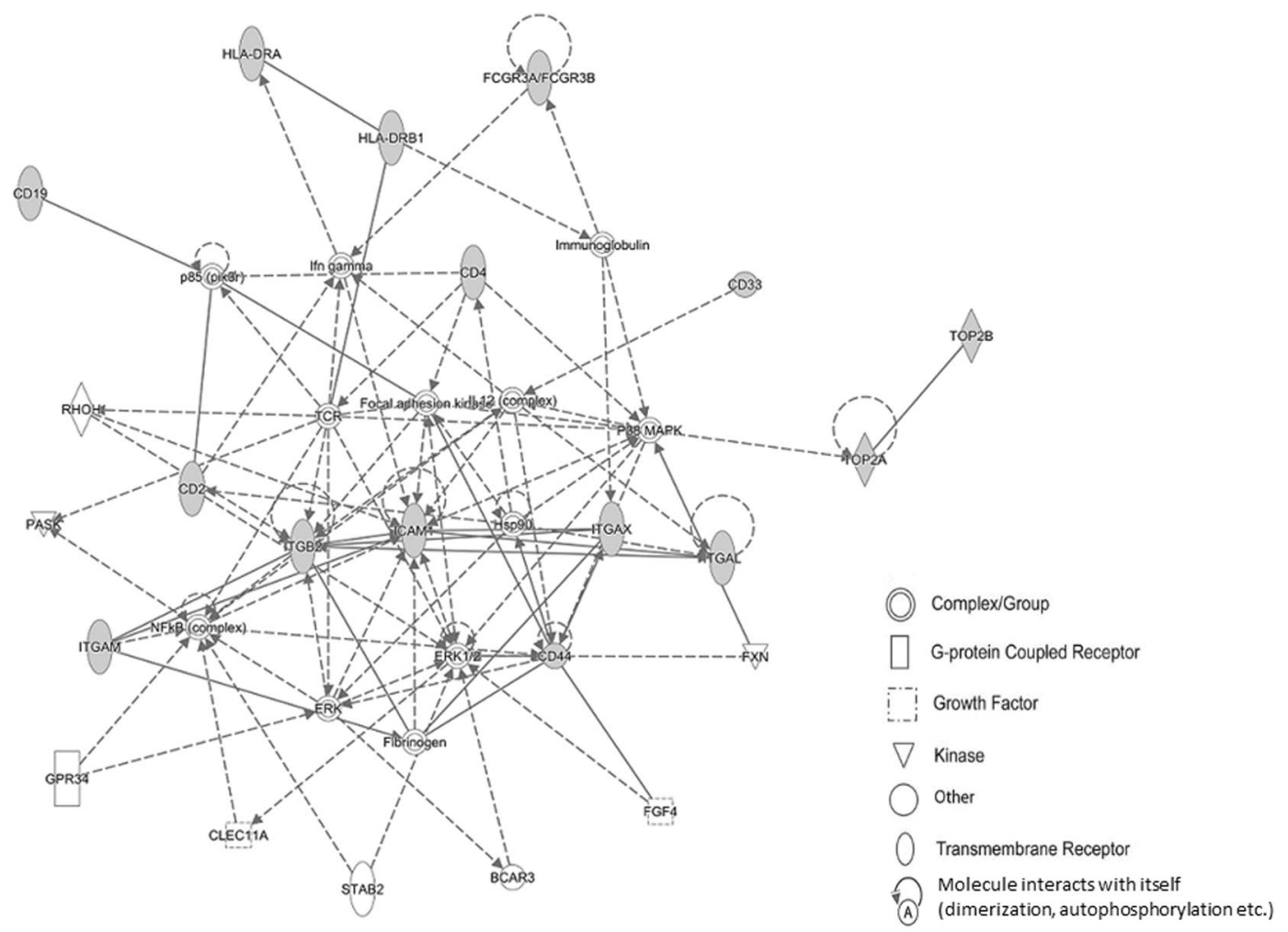

Figure 2. Ingenuity pathway analysis of drug pharmacodynamic targets, associated immunophenotype markers and topo II. The (-) and (---) lines in network represent direct and in indirect interaction, respectively. Gene ID is identified in the network symbols and symbol key describes biological relevance. Gray fill color in symbols identifies focus genes from the dataset.

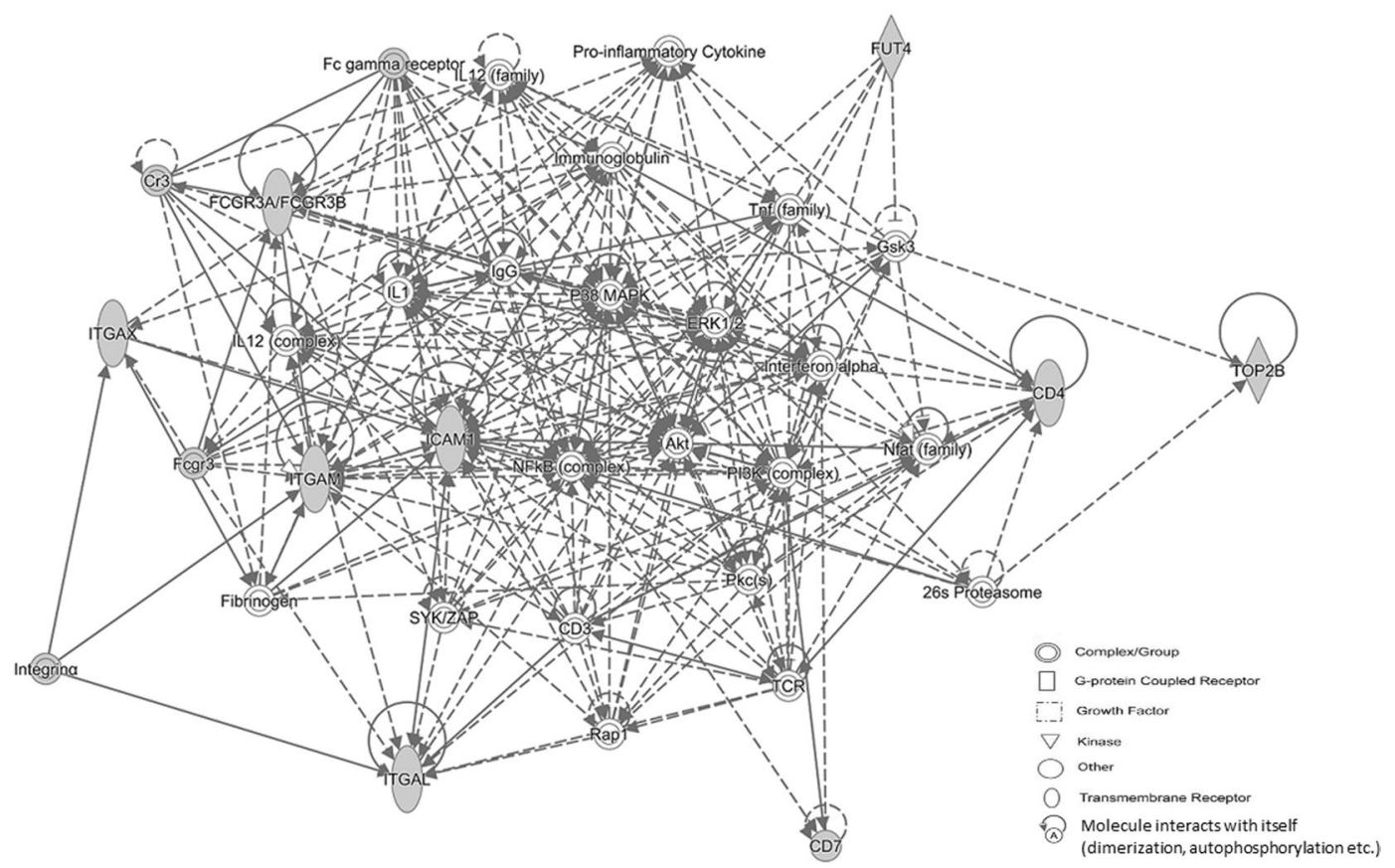

Figure 3. Ingenuity pathway analysis of topo II $\beta$ and significantly associated immunophenotype markers. The $(-)$ and (--) lines in network represent direct and in indirect interaction, respectively. Gene ID is identified in the network symbols and symbol key describes biological relevance. Gray fill color in symbols identifies focus genes from the dataset. 
expression of 40 was considered right-censored. Topo II $\alpha$ expression was censored on four observations and topo II $\beta$ expression on three observations.

Clinical data (age, sex, race/ethnicity, secondary versus de novo AML onset, FAB classification, cytogenetics, marrow and peripheral blood blast percentages, WBC and PLT counts, and hemoglobin) and treatment outcomes were collected and evaluated per standard SWOG procedures as part of the clinical trials on which the patients participated. Complete response (CR) and resistant disease (RD) were defined by standard criteria ${ }^{35}$. Overall survival (OS) was measured from date of study entry until death from any cause, with observation censored at the date of last contact for patients last known to be alive. Relapse-free survival (RFS) was measured from the date of achieving CR until relapse or death from any cause, with observation censored at the date of last contact for patients last known to be alive without report of AML relapse. Linear regression models were used to examine the effects of patient characteristics and immunophenotype on expression. The effects of expression and other patient characteristics on treatment outcomes were investigated using logistic (CR, RD) and proportional hazards (OS, RFS) regression analyses.

Multivariate analyses for the outcomes of OS, RFS, RD, and CR accounted for clinical and immunophenotype characteristics. Additional factors were excluded as needed to fit each model: expression of CD2, CD19, CD56, and HLA-DR on CR; expression of CD2, CD19, CD54, CD56, and HLA-DR on RD; expression of CD54 on OS; and expression of CD2, CD4, CD11a, CD16, CD19, C33, CD44, CD54, CD56, and HLA-DR on RFS.

Ingenuity pathway analysis (Qiagen Inc.) of target molecules identified for significant association with topo II $\alpha / \beta$ isoform expression was carried out using version 46901286 (11-21-2018). Ingenuity pathway analysis was carried out using: (a) drug pharmacodynamic targets, associated immunophenotype markers and topo II $\alpha / \beta$; and (b) topo II $\beta$ and significantly associated immunophenotype markers.

Received: 11 September 2019; Accepted: 12 March 2020;

Published online: 26 March 2020

\section{References}

1. Tallman, M. S. New strategies for the treatment of acute myeloid leukemia including antibodies and other novel agents. Hematology Am Soc Hematol Educ Program. 143-150 (2005).

2. Tallman, M. S., Gilliland, D. G. \& Rowe, J. M. Drug therapy for acute myeloid leukemia. Blood. 106, 1154-1163 (2005). Erratum in: Blood. 106, 2243 (2005).

3. Fordham, S. E., Matheson, E. C., Scott, K., Irving, J. A. E. \& Allan, J. M. DNA mismatch repair status affects cellular response to ara-c and other anti-leukemic nucleoside analogues. Leukemia. 25, 1046-1049 (2011).

4. Chikamori, K. et al. DNA topoisomerase II enzymes as molecular targets for cancer chemotherapy. Curr Cancer Drug Targets. 10, 758-771 (2010).

5. Ganapathi, R. N. \& Ganapathi, M. K. Mechanisms regulating resistance to inhibitors of topoisomerase II. Front. Pharmacol. 4, 89 (2013).

6. Li, T. K. \& Liu, L. F. Tumor cell death induced by topoisomerase-targeting drugs. Annu Rev Pharmacol. 41, 53-57 (2001).

7. Wang, J. C. Cellular roles of DNA topoisomerases: a molecular perspective. Nat Rev Mol Cell Biol. 3, 430-440 (2002).

8. Woessner, R. D., Mattern, M. R., Mirabelli, C. K., Johnson, R. K. \& Drake, F. H. Proliferation- and cell cycle-dependent differences in expression of the 170 kilodalton and 180 kilodalton forms of topoisomerase II in NIH-3T3 cells. Cell Growth Differ. 2, 209-214 (1991).

9. Nitiss, J. L. DNA topoisomerase II and its growing repertoire of biological functions. Nat Rev Can. 9, 327-337 (2009).

10. Turley, H. et al. The distribution and expression of the two isoforms of DNA topoisomerase II in normal and neoplastic human tissues. Brit J Cancer. 75, 1340-1346 (1997).

11. Capranico, G., Tinelli, S., Austin, C. A., Fisher, M. L. \& Zunino, F. Different patterns of gene expression of topoisomerase II isoforms in differentiated tissues during murine development. Biochim Biophys Acta. 1132, 43-48 (1992).

12. Chikamori, K. et al. Downregulation of topoisomerase II $\beta$ in myeloid leukemia cell lines leads to activation of apoptosis following all-trans retinoic acid-induced differentiation/growth arrest. Leukemia. 20, 1809-1818 (2006).

13. Kozuki, T. et al. Roles of the C-terminal domains of topoisomerase II $\alpha$ and topoisomerase II $\beta$ in regulation of the decatenation checkpoint. Nucleic Acids Res. 45, 5995-6010 (2017).

14. Di, L. A. et al. HER2/TOP2A Meta-analysis Study Group, HER2 and TOP2A as predictive markers for anthracycline-containing chemotherapy regimens as adjuvant treatment of breast cancer: a meta-analysis of individual patient data. Lancet Oncol. 12, 1134-1142(2011).

15. Kaufmann, S. H. et al. Topoisomerase II levels and drug sensitivity in adult acute myelogenous leukemia. Blood. 83, 517-530 (1994).

16. McKenna, S. L., West, R. R., Whittaker, J. A., Padua, R. A. \& Holmes, J. A. Topoisomerse II o expression in Acute Myeloid Leukaemia and its relationship to clinical outcome. Leukemia. 8, 1498-1502 (1994).

17. Zhou, R. et al. Real-time RT-PCR for the determination of topoisomerase II mRNA level in leukaemic cells. Leuk Res. 26, 487-494 (2002).

18. Chen, C. C. et al. Prognostic significance of $\beta$-catenin and topoisomerase II $\alpha$ in de novo acute myeloid leukemia. Am J Hematol. 84, 87-92 (2009)

19. Tina, E., Prenkert, M., Höglund, M., Paul, C. \& Tidefelt, U. Topoisomerase II $\alpha$ expression in acute myeloid leukaemia cells that survive after exposure to daunorubicin or ara-C. Oncol Rep. 22, 1527-1531 (2009).

20. Herzog, C. E., Holmes, K. A., Tuschong, L. M., Ganapathi, R. \& Zwelling, L. A. Absence of topoisomerase IIbeta in an amsacrineresistant human leukemia cell line with mutant topoisomerase IIalpha. Cancer Res. 58, 5298-3000 (1998).

21. Hermanson, D. L., Das, S. G., Li, Y. \& Xing, C. Overexpression of Mcl-1 confers multidrug resistance, whereas topoisomerase II $\beta$ downregulation introduces mitoxantrone-specific drug resistance in acute myeloid leukemia. Mol Pharmacol. 84, 236-243 (2013).

22. McNamara, S., Wang, H., Hanna, N. \& Miller, W. H. Jr. Topoisomerase II $\beta$ negatively modulates retinoic acid receptor $\alpha$ function: a novel mechanism of retinoic acid resistance. Mol Cell Biol. 28, 2066-2077 (2008).

23. Mistry, A. et al. DNA topoisomerase II in therapy-related acute promyelocytic leukemia. N Engl J Med. 352, 1529-1538 (2005).

24. Gieseler, F. et al. Topoisomerase II activities in AML and their correlation with cellular sensitivity to anthracyclines and epipodophyllotoxines. Leukemia. 10, 1177-1180 (1996).

25. Legrand, O. et al. The immunophenotype of 177 adults with acute myeloid leukemia: proposal of a prognostic score. Blood. 96, 870-877 (2000)

26. Pagano, L. et al. Immunophenotypic analysis in 119 patients with acute myeloid leukemia following a previous malignancy: a comparison with the immunophenotype of 231 de novo AML. Haematologica. 88, 225-227 (2003).

27. Ossenkoppele, G. J. et al. Review of the relevance of aberrant antigen expression by flow cytometry in myeloid neoplasms. $\mathrm{Br} J$ Haematol. 153, 421-436 (2011). 
28. Costa, A. F. O. et al. Role of new Immunophenotypic Markers on Prognostic and Overall Survival of Acute Myeloid Leukemia: a Systematic Review and Meta-Analysis. Sci Rep. 7, 4138 (2017).

29. van Solinge, T. S. et al. The interference of genetic associations in establishing the prognostic value of the immunophenotype in acute myeloid leukemia. Cytometry B Clin Cytom. 94, 151-158 (2018).

30. Song, J. N. et al. High TOP2B/TOP2A expression ratio at diagnosis correlates with favourable outcome for standard chemotherapy in acute myeloid leukemia. Brit J Cancer. 107, 108-115 (2012).

31. Bradstock, K. F. et al. Australasian Leukaemia \& Lymphoma Group, Idarubicin Dose Escalation During Consolidation Therapy for Adult Acute Myeloid Leukemia. J Clin.Oncol. 35, 1678-1685 (2017).

32. Lee, J. H. et al. Cooperative Study Group A for Hematology. Prospective Randomized Comparison of Idarubicin and High-Dose Daunorubicin in Induction Chemotherapy for Newly Diagnosed Acute Myeloid Leukemia. J Clin Oncol. 35, 2754-2763 (2017).

33. Patel, C. et al. Multidrug resistance in elapsed acute myeloid leukemia: evidence of biological heterogeneity. Cancer. 115, 3076-3083 (2013).

34. Horibata, S. et al. Heterogeneity in refractory acute myeloid leukemia. Proc Natl Acad Sci USA 116, 10494-10503 (2019).

35. Cheson, B. D. et al. Revised recommendations of the International Working Group for Diagnosis, Standardization of Response Criteria, Treatment Outcomes, and Reporting Standards for Therapeutic Trials in Acute Myeloid Leukemia. J Clin Oncol. 21, 4642-4649 (2003). Erratum in: J Clin Oncol. 22, 576 (2004).

\section{Acknowledgements}

Supported in part by the following National Institutes of Health, National Cancer Institute grant awards: CA180888, CA180819, CA233328, CA004919, CA196175, and legacy grants CA012213, CA20319 and CA073590; and part by Amgen, Inc. and Gene Expression \& Genotyping Core Facility of the Case Comprehensive Cancer Center (P30CA043703). The content is solely the responsibility of the authors and does not necessarily represent the official views of the National Institutes of Health.

\section{Author contributions}

A.P.M., M.K.G., A.S.A. and R.N.G. conceptualized the project. A.P.M., E.R.K., M.K.G., A.S.A. and R.N.G. contributed to the design and methodology for the experiments performed. S.M. and M.O. performed formal (statistical) analysis. A.P.M., S.M., M.O., M.K.G., A.S.A. and R.N.G. wrote the original first draft of the manuscript. A.P.M., S.M., C.L.W., E.R.K., J.E.G., S.H.P. A.F.L., M.O., F.R.A., J.P.R., M.K.G., A.S.A., and R.N.G. were involved in review and editing of the manuscript.

\section{Competing interests}

The authors declare no competing interests.

\section{Additional information}

Correspondence and requests for materials should be addressed to A.S.A. or R.N.G.

Reprints and permissions information is available at www.nature.com/reprints.

Publisher's note Springer Nature remains neutral with regard to jurisdictional claims in published maps and institutional affiliations.

Open Access This article is licensed under a Creative Commons Attribution 4.0 International License, which permits use, sharing, adaptation, distribution and reproduction in any medium or format, as long as you give appropriate credit to the original author(s) and the source, provide a link to the Creative Commons license, and indicate if changes were made. The images or other third party material in this article are included in the article's Creative Commons license, unless indicated otherwise in a credit line to the material. If material is not included in the article's Creative Commons license and your intended use is not permitted by statutory regulation or exceeds the permitted use, you will need to obtain permission directly from the copyright holder. To view a copy of this license, visit http://creativecommons.org/licenses/by/4.0/.

(c) The Author(s) 2020 